\title{
A Note on the Wick Integral with Respect to Fractional Brownian Sheet
}

\author{
Joon Hee Rhee ${ }^{a}$, Yoon Tae Kim ${ }^{1, b}$ \\ ${ }^{a}$ Department of Business and Administration, Soong-Sil University \\ ${ }^{b}$ Department of Statistics, Hallym University
}

\begin{abstract}
By using the white noise theory for fractional Brownian sheet, we give new representations of the Wick integrals of various types with respect to fractional Brownian sheet with Hurst parameters $H_{1}, H_{2} \in(0,1)$.

Keywords: Fractional Brownian sheet, white noise theory, stochastic line integral, wick integrals, wick product.
\end{abstract}

\section{Introduction and Notations}

Recall that a real-valued fractional Brownian sheet(fBs) $B^{H}$ with Hurst parameter $H=\left(H_{1}, H_{2}\right)$, $H_{1}, H_{2} \in(0,1)$, is a centered Gaussian random fields with covariance

$$
\mathbb{E}\left[B^{H}(a) B^{H}(a)\right]=\prod_{i=1}^{2} \frac{1}{2}\left(\left|a_{i}\right|^{2 H_{i}}+\left|b_{i}\right|^{2 H_{i}}-\left|a_{i}-b_{i}\right|^{2 H_{i}}\right),
$$

where $a=\left(a_{1}, a_{2}\right), b=\left(b_{1}, b_{2}\right) \in R^{2}$. The elementary theory of stochastic calculus for fBs has recently been developed by several authors (see Kim, 2006, 2009; Kim and Jeon, 2006; Kim et al., 2008, 2009; Kim and Rhee, 2008; Kim and Park, 2009; Tudor and Viens, 2003). Among them, Kim and Jeon (2006) define the following Wick integrals with respect to fBs and derive an Itô formula for $\mathrm{fBs}$ by using the white noise theory for $\mathrm{fBs}$ : for $z=\left(z_{1}, z_{2}\right) \in R_{+}^{2}=[0, \infty) \times[0, \infty)$

$$
\begin{aligned}
& \int_{R_{z}} \alpha(a) d B^{H}(a), \quad \int_{R_{z} \unlhd, R_{z}} \beta(a, b) d B^{H}(a) d B^{H}(b), \\
& \int_{R_{z} \unlhd_{1} R_{z}} \beta(a, b) d a d B^{H}(b), \quad \int_{R_{z} \unlhd_{1} R_{z}} \beta(a, b) d B^{H}(b) d b,
\end{aligned}
$$

where $R_{z}=\left[0, z_{1}\right] \times\left[0, z_{2}\right]$ and the set $R_{z} \unlhd_{1} R_{z}$ will be defined below.

In this paper, we prove that the Wick integral of the process $\partial_{1} B^{H}(a) \diamond \partial_{2} B^{H}(a)$ exists, where the notation $\diamond$ denote the Wick product. By using this integral, we give a new representation of the second Wick integral in (1.1). On the other hand, for new representations of the third and fourth Wick integrals in (1.1), we use stochastic line integrals with respect to $\mathrm{fBs}$, being defined in this paper.

We give some notations that are used throughout the paper. Let $a=\left(a_{1}, a_{2}\right)$ and $b=\left(b_{1}, b_{2}\right)$ be two points in $R^{2}$.

\footnotetext{
${ }^{1}$ Corresponding author: Professor, Department of Statistics, Hallym University, Chuncheon, Gangwon-do 200-702, Korea. E-mail: ytkim@hallym.ac.kr
} 
- The notation $a \leq b$ will denote the condition $a_{1} \leq b_{1}$ and $a_{2} \leq b_{2}$.

- The notation $a \unlhd_{1} b$ will denote the condition $a_{1} \leq b_{1}$ and $a_{2} \geq b_{2}$.

- The notation $a * b$ will denote the point $\left(a_{1}, b_{2}\right)$.

- For $a \leq b$, the notation $R_{[a, b]}$ will denote the rectangle $[a, b]$ and $R_{[0, b]}=R_{b}$.

- For $x=\left(x_{1}, \ldots, x_{n}\right) \in \mathbb{R}^{n}$ and $\alpha=\left(\alpha_{1}, \ldots, \alpha_{n}\right) \in \mathbb{R}^{n}$, let us set $x^{\alpha}=\prod_{i=1}^{n} x_{i}^{\alpha}$ and $d x^{\alpha}=\prod_{i=1}^{n} d x_{i}^{\alpha}$.

- The notation $R_{z} \unlhd_{1} R_{z}$ will denote the set $\left\{(a, b) \in R_{z} \times R_{z}: b \unlhd_{1} a\right\}$.

\section{Preliminaries}

We recall the white noise theory for $\mathrm{fBs}$, given in $\mathrm{Hu}$ et al. (1996) (see Biagini et al. (2004) and Elliott and van der Hoek (2003) for fractional Brownian motion), to be necessary for our works. Let $\mathcal{S}\left(R^{2}\right)$ be the Schwartz space of rapidly decreasing smooth functions on $R^{2}$. We consider the white noise space $(\Omega, \mathbf{F}, \mathbb{P})$ as the underlying probability space, i.e, $\Omega:=\mathcal{S}^{\prime}\left(R^{2}\right)$ is the space of tempered distributions and $\mathbb{P}$ is an unique probability measure such that

$$
\int_{S^{\prime}\left(R^{2}\right)} e^{i<\omega, f>} d \mathbb{P}(\omega)=e^{-\left(\frac{1}{2}\right)\|f\|_{L^{2}\left(R^{2}\right)}^{2}}, \quad \text { for } f \in \mathcal{S}\left(R^{2}\right) .
$$

Then we have the isometry $\mathbb{E}[<\cdot f\rangle\langle\cdot, g\rangle]=(f, g)_{L^{2}\left(R^{2}\right)}$, and using this we can extend $\langle\cdot, f>$ to $f \in L^{2}\left(R^{2}\right)$. For $a, b \in R^{2}$, we define $\mathbf{1}_{(a, b)}(x)=\prod_{i=1}^{2} \mathbf{1}_{\left(a_{i}, b_{i}\right)}\left(x_{i}\right)$ for $x=\left(x_{1}, x_{2}\right)$, where the indicator function $\mathbf{1}_{\left(a_{i}, b_{i}\right)}\left(x_{i}\right)$ is given by

$$
\mathbf{1}_{\left(a_{i}, b_{i}\right)}\left(x_{i}\right)=\left\{\begin{aligned}
1, & \text { for } a_{i} \leq x_{i} \leq b_{i} \\
-1, & \text { for } b_{i} \leq x_{i} \leq a_{i}, \\
0, & \text { otherwise. }
\end{aligned}\right.
$$

For $f \in S\left(R^{2}\right)$, we define an operator $I_{H_{i}} f: R^{2} \rightarrow R, i=1,2$, by

$$
I_{H_{i}} f(x)= \begin{cases}C_{H_{i}} \int_{R} \frac{f\left(x+u \epsilon_{i}\right)}{|u|^{\left(\frac{3}{2}\right)-H_{i}}} d u, & \text { for } \frac{1}{2}<H_{i}<1, \\ f, & \text { for } H_{i}=\frac{1}{2}, \\ C_{H_{i}} \int_{R} \frac{f\left(x-u \epsilon_{i}\right)-f(x)}{|u|^{\left(\frac{3}{2}\right)-H_{i}}} d u, & \text { for } 0<H_{i}<\frac{1}{2},\end{cases}
$$

where $\epsilon_{1}=(1,0), \epsilon_{2}=(0,1)$ and

$$
C_{H_{i}}=\frac{\sin \left(\pi H_{i}\right) \Gamma\left(2 H_{i}+1\right)}{2 \Gamma\left(H_{i}-(1 / 2)\right) \cos \left((\pi / 2)\left(H_{i}-(1 / 2)\right)\right.} .
$$

Let $I_{H} f(x)=I_{H_{1}}\left(I_{H_{2}}\right) f(x)$. Then a continuous version of $\left\langle\cdot, I_{H} \mathbf{1}_{(0, a)}\right\rangle$ is fBs with arbitrary Hurst parameters $H=\left(H_{1}, H_{2}\right), H_{1}, H_{2} \in(0,1)$ on $(\Omega, \mathbf{F}, \mathbb{P})$.

Let $\mathbf{H}_{n}(x)$ and $h_{n}, n=0,1, \ldots$, be the $n^{\text {th }}$ Hermite polynomial and the $n^{\text {th }}$ Hermite function respectively. For $\alpha=\left(\alpha_{1}, \alpha_{2}\right) \in \mathbb{N}^{2}$ (with $\mathbb{N}=\{1,2, \ldots\}$ ), let us set $\mathbf{e}_{\alpha}\left(x_{1}, x_{2}\right)=\prod_{i=1}^{2} h_{\alpha_{i}}\left(x_{i}\right)$. Denote by $\mathbf{A}$ the set of all finite sequences $\mathbf{a}=\left(a_{1}, a_{2}, \ldots, a_{m}\right)$ with $a_{i} \in\{0\} \cup \mathbb{N}, m=1,2, \ldots$. For $\mathbf{a} \in \mathbf{A}$, we set 
$\mathbf{a} !=\prod_{i=1}^{\infty} a_{i} !$ and $|\mathbf{a}|=\sum_{i=1}^{\infty} a_{i}$. Let $\alpha^{(i)}, i=1,2, \ldots$, be a fixed ordering of $\mathbb{N}^{2}$ such that for $i<j$, $\left|\alpha^{(i)}\right| \leq\left|\alpha^{(j)}\right|$. With these notations, we define

$$
\mathbf{H}_{\mathbf{a}}(\omega)=\prod_{i=1}^{\infty} \mathbf{H}_{a_{i}}\left(<\omega, \mathbf{e}_{\alpha^{(i)}}>\right) .
$$

We recall the following chaos expansion theorem.

Theorem 1. Let $F \in \mathbb{L}^{2}:=L^{2}(\Omega, \mathbf{F}, \mathbb{P})$. Then there exist constants $c_{\mathbf{a}} \in \mathbb{R}$ for $\mathbf{a} \in \mathbf{A}$ such that

$$
F(\omega)=\sum_{\mathbf{a} \in \mathbf{A}} c_{\mathbf{a}} \mathbf{H}_{\mathbf{a}}(\omega) \quad \text { limit in } \mathbb{L}^{2}
$$

Furthermore, we have the isometry $\|F\|_{\mathbb{L}^{2}}^{2}=\sum_{\mathbf{a} \in \mathbf{A}} \mathbf{a} ! c_{\mathbf{a}}^{2}$.

Let $\mathbb{Z}$ be the set of all integers. For $p \in \mathbb{Z}$ and $F$ given in (2.2), we define the norm $\|F\|_{p}^{2}=$ $\sum_{\mathbf{a} \in \mathbf{A}} c_{\mathbf{a}}^{2} \mathbf{a} !(2 \mathbb{N})^{p \mathbf{a}}$, where $(2 \mathbb{N})^{\mathbf{a}}=\prod_{l=1}^{m}(2 l)^{a_{l}}$ with $\mathbf{a}=\left(a_{1}, \ldots, a_{m}\right)$. If $p \in \mathbb{N}$, we define the space $(\mathbb{S})_{p}=\left\{F \in \mathbb{L}^{2}:\|F\|_{p}^{2}<\infty\right\}$ and endow $(\mathbb{S})_{p}$ with the norm $\|\cdot\|_{p}$. Also define $(\mathbb{S})_{-p}=\left\{F \in \mathbb{L}^{2}\right.$ : $\left.\|F\|_{-p}^{2}<\infty\right\}$.

Definition 1. (1) The projective limit of the spaces $(\mathbb{S})_{p}, p \in \mathbb{N}$, is called the space of the stochastic test functions and denoted by $(\mathbb{S})$. (2) The inductive limit of the spaces $(\mathbb{S})_{-p}, p \in \mathbb{N}$, is called the space of stochastic distributions and denoted by $(\mathbb{S})^{*}$. (3) For $F(\omega)=\sum_{\mathbf{a} \in \mathbf{A}} c_{\mathbf{a}} \mathbf{H}_{\mathbf{a}}(\omega)$ and $G(\omega)=$ $\sum_{\mathbf{b} \in \mathbf{A}} d_{\mathbf{b}} \mathbf{H}_{\mathbf{b}}(\omega)$, define the Wick product $(F \diamond G)(\omega)=\sum_{\mathbf{a}, \mathbf{b} \in \mathbf{A}} c_{\mathbf{a}} d_{\mathbf{b}} \mathbf{H}_{\mathbf{a}+\mathbf{b}}(\omega)$.

We first note that if $f \in \mathcal{S}\left(R^{2}\right)$, then $I_{H} f \in L^{2}\left(R^{2}\right)$. Furthermore, if $f, g \in \mathcal{S}\left(R^{2}\right)$, then $\left(f, I_{H} g\right)_{L^{2}\left(R^{2}\right)}=$ $\left(I_{H} f, g\right)_{L^{2}\left(R^{2}\right)}$. Hence with the above notations, the chaos expansion of $\mathrm{fBs}$ is given by

$$
B^{H}(a, \omega)=\sum_{l=1}^{\infty}\left(I_{H} \mathbf{e}_{\alpha^{(l)}}, \mathbf{1}_{(0, a)}\right)_{L^{2}\left(R^{2}\right)} \mathbf{H}_{\epsilon_{l}}(\omega),
$$

where $\epsilon_{l}=(0, \ldots, 0,1,0, \ldots, 0), l^{\text {th }}$ unit vector.

The two-parameter fractional white noise $W^{H}(a, \omega)$ is defined by the derivative in $(\mathbb{S})^{*}$ of $B^{H}(a, \omega)$ :

$$
W^{H}(a, \omega)=\frac{\partial^{2} B(a, \omega)}{\partial a_{1} \partial a_{2}}=\sum_{l=1}^{\infty}\left(I_{H} \mathbf{e}_{\alpha^{(l)}}\right)(a) \mathbf{H}_{\epsilon_{l}}(\omega) .
$$

\section{Wick Integrals of Various Types}

First we give the definition on $(\mathbb{S})^{*}$-valued surface integrals for multi-parameter case.

Definition 2. Let $Z: \mathbb{R}^{n} \rightarrow(\mathbb{S})^{*}$ be a given function satisfying

$$
\int_{R^{n}}\left|\ll Z(\mathbf{t}), \varphi \gg_{(\mathbb{S})^{*},(\mathbb{S})}\right| d \mathbf{t}<\infty, \text { for all } \varphi \in(\mathbb{S}),
$$

where $\ll, \cdot \gg_{(\mathbb{S})^{*},(\mathbb{S})}$ is the bi-pairing $(\mathbb{S})^{*}$ and $(\mathbb{S})$, and $\mathbf{t}=\left(t_{1}, \ldots, t_{n}\right) \in R^{n}$. Then we define $(\mathbb{S})^{*}-$ valued integral $\int_{R^{n}} Z(\mathbf{t}) d \mathbf{t}$ to be the unique element of $(\mathbb{S})^{*}$ such that for all $\varphi \in(\mathbb{S})$,

$$
\ll \int_{R^{n}} Z(\mathbf{t}) d \mathbf{t}, \varphi \gg_{(\mathbb{S})^{*},(\mathbb{S})}=\int_{R^{n}} \ll Z(\mathbf{t}), \varphi \gg_{(\mathbb{S})^{*},(\mathbb{S})} d \mathbf{t} .
$$


If (3.1) holds, we then say that $Z$ is integrable in $(\mathbb{S})^{*}$.

For $\alpha^{(l)}=\left(\alpha_{1}^{(l)}, \alpha_{2}^{(l)}\right)$, we define two partial fractional white noises:

$$
\begin{aligned}
& \partial_{1} B^{H}(a, \omega)=\sum_{l=1}^{\infty}\left(I_{H_{1}} h_{\alpha_{1}^{(l)}}\right)\left(a_{1}\right)\left(I_{H_{2}} h_{\alpha_{2}^{(n)}}, \mathbf{1}_{\left(0, a_{2}\right)}\right)_{L^{2}(R)} \mathbf{H}_{\epsilon_{l}}(\omega), \\
& \partial_{2} B^{H}(a, \omega)=\sum_{l=1}^{\infty}\left(I_{H_{1}} h_{\alpha_{1}^{(n)}}, \mathbf{1}_{\left(0, a_{1}\right)}\right)_{L^{2}(R)}\left(I_{H_{2}} h_{\alpha_{2}^{(l)}}\right)\left(a_{2}\right) \mathbf{H}_{\epsilon_{l}}(\omega) .
\end{aligned}
$$

Then it is easily shown that the noises, defined by (3.2) and (3.3), satisfy that $\partial_{1} B^{H}(a) \in(\mathbb{S})^{*}$ and $\partial_{2} B^{H}(a) \in(\mathbb{S})^{*}$ for each $a \in R^{2}$.

Definition 3. Let $\gamma(\sigma)=\left(\gamma_{1}(\sigma), \gamma_{2}(\sigma)\right)$ be a smooth curve on $\sigma \in[0,1]$ and $Z: R^{2} \rightarrow(\mathbb{S})^{*}$ be a given function such that for $i=1,2$ and for all $\varphi \in(\mathbb{S})$,

$$
\int_{0}^{1}\left|\ll Z(\gamma(\sigma)) \diamond \partial_{i} B^{H}(\gamma(\sigma)), \varphi \gg_{(\mathbb{S})^{*},(\mathbb{S})} \gamma_{i}^{\prime}(\sigma)\right| d \sigma<\infty
$$

Then we define $(\mathbb{S})^{*}$-valued line integral $\int_{y} Z \partial_{i} B^{H}$ to be the unique element of $(\mathbb{S})^{*}$ such that for all $\varphi \in(\mathbb{S})$,

$$
\ll \int_{\gamma} Z \partial_{i} B^{H}, \varphi \gg_{(\mathbb{S})^{*},(\mathbb{S})}=\int_{\gamma} \ll Z(a) \diamond \partial_{i} B^{H}(a), \varphi \gg_{(\mathbb{S})^{*},(\mathbb{S})} d a_{i}, \quad i=1,2 .
$$

Remark 1. We may consider the Wick integrals $\int_{\gamma} Z \partial_{i} B^{H}, i=1,2$, as the line integral of the stochastic differential 1-forms $Z(a) \diamond \partial_{i} B^{H} d a_{i}$ in $R^{2}$. Let $h_{z}$ and $v_{z}$ be the horizontal line segment connecting a point $z \in[0, T]$ with $y$-axis and the vertical line segment connecting a point $z \in[0, T]$ with $x$-axis, respectively. Then the stochastic line integrals $\int_{h_{z}} Z \partial_{1} B^{H}$ and $\int_{v_{z}} Z \partial_{2} B^{H}$ can be represented as

$$
\begin{aligned}
& \int_{h_{z}} Z \partial_{1} B^{H}=\int_{0}^{z_{1}} Z(a * z) \diamond \partial_{1} B^{H}(a * z) d a_{1} \\
& \int_{v_{z}} Z \partial_{2} B^{H}=\int_{0}^{z_{1}} Z(z * a) \diamond \partial_{1} B^{H}(z * a) d a_{2},
\end{aligned}
$$

respectively. We refer to Cairoli and Walsh (1975) for standard Brownian sheet.

The following lemma is the direct extension of Lemma 2.5.6 in Holden et al. (1996) to twoparameter processes.

Lemma 1. If $Z(a): R^{2} \rightarrow(\mathbb{S})^{*}$ has an expansion $Z(a)=\sum_{\mathbf{a} \in \mathbf{A}} c_{\mathbf{a}}(a) \mathbf{H}_{\mathbf{a}}(\omega)$, where

$$
\sum_{\mathbf{a} \in \mathbf{A}} \mathbf{a} !\left\|c_{\mathbf{a}}\right\|_{L^{1}\left(R^{2}\right)}(2 \mathbb{N})^{-p \mathbf{a}}<\infty, \text { for some } p>0 .
$$

Then $Z(a)$ is da-integrable in $(\mathbb{S})^{*}$ and

$$
\int_{R^{2}} Z(a) d a=\sum_{\mathbf{a} \in \mathbf{A}} \int_{R^{2}} c_{\mathbf{a}}(a) d a \mathbf{H}_{\mathbf{a}}(\omega)
$$

Now we show that the Wick product of two partial fractional white noises, given in (3.2) and (3.3), is integrable in $(\mathbb{S})^{*}$. 
Lemma 2. A process $\partial_{1} B^{H}(a) \diamond \partial_{2} B^{H}(a)$ is da-integrable in $(\mathbb{S})^{*}$ and

$$
\begin{aligned}
\int_{R_{z}} \partial_{1} B^{H}(a) \diamond \partial_{2} B^{H}(a) d a= & \left.\sum_{l, k=1}^{\infty} \int_{R_{z}}\left(I_{H_{1}} h_{\alpha_{1}^{(n)}}, \mathbf{1}_{\left(0, a_{1}\right)}\right)_{L^{2}(R)}\left(I_{H_{2}} h_{\alpha_{2}^{(k)}}, \mathbf{1}_{\left(0, a_{2}\right)}\right)\right)_{L^{2}(R)} \\
& \times\left(I_{H_{1}} h_{\alpha_{1}^{(k)}}\right)\left(a_{1}\right)\left(I_{H_{2}} h_{\alpha_{2}^{(i)}}\right)\left(a_{2}\right) d a \mathbf{H}_{\epsilon_{l}+\epsilon_{k}}(\omega) .
\end{aligned}
$$

Proof: Since $\partial_{1} B^{H}(a) \diamond \partial_{2} B^{H}(a) \in(\mathbb{S})^{*}$ and

$$
\begin{aligned}
\partial_{1} B^{H}(a) \diamond \partial_{2} B^{H}(a)= & \sum_{\mathbf{a} \in \mathbf{A}} \sum_{\substack{l i k \\
\epsilon_{1}+\epsilon_{k}=\mathbf{a}}}\left(I_{H_{1}} h_{\alpha_{1}^{(i)}}, \mathbf{1}_{\left(0, a_{1}\right)}\right)_{L^{2}(R)}\left(I_{H_{2}} h_{\alpha_{2}^{(k)}}, \mathbf{1}_{\left(0, a_{2}\right)}\right)_{L^{2}(R)} \\
& \times\left(I_{H_{1}} h_{\alpha_{1}^{(k)}}\right)\left(a_{1}\right)\left(I_{H_{2}} h_{\alpha_{2}^{(l)}}\right)\left(a_{2}\right) \mathbf{1}_{R_{z}}(a) \mathbf{H}_{\mathbf{a}}(\omega),
\end{aligned}
$$

the result follows from Lemma 1 if we prove that for some $p>0$,

$$
\sum_{\mathbf{a} \in \mathbf{A}} \mathbf{a} ! \xi(\mathbf{a})(2 \mathbb{N})^{-p \mathbf{a}}<\infty
$$

where

$$
\left.\xi(\mathbf{a})=\| \sum_{\substack{i, k \\ \epsilon_{1}+k_{k}=\mathbf{a}}}\left(I_{H_{1}} h_{\alpha_{1}^{(i)}}, \mathbf{1}_{\left(0, a_{1}\right)}\right)\right)_{L^{2}(R)}\left(I_{H_{2}} h_{\alpha_{2}^{(k)}}, \mathbf{1}_{\left(0, a_{2}\right)}\right)_{L^{2}(R)} \times\left(I_{H_{1}} h_{\alpha_{1}^{(k)}}\right)\left(a_{1}\right)\left(I_{H_{2}} h_{\alpha_{2}^{(l)}}\right)\left(a_{2}\right) \mathbf{1}_{R_{z}}(a) \|_{L^{1}\left(R^{2}\right)}^{2} .
$$

Since $\left|I_{H_{i}} h_{\alpha_{i}^{(k)}}\left(a_{i}\right)\right| \leq C k^{2 / 3-H_{i} / 2} \leq C k^{2 / 3}, i=1,2$, for some $C>0$ and $k \geq 1$ (see e.g. Elliott and van der Hoek, 2003),

$$
\int_{R_{z}}\left|I_{H_{1}} \otimes I_{H_{2}}\left(h_{\alpha_{1}^{(k)}} \otimes h_{\alpha_{2}^{(l)}}\right)(a) \int_{R_{a}} I_{H_{1}} \otimes I_{H_{2}}\left(h_{\alpha_{1}^{(k)}} \otimes h_{\alpha_{2}^{(n)}}\right)(b) d b\right| d a \leq C\left(z_{1} z_{2}\right)^{2}(k l)^{\frac{4}{3}} .
$$

The above estimate gives

$$
\xi(\mathbf{a}) \leq C\left(z_{1} z_{2}\right)^{2}\left(\sum_{\substack{l, k \\ \epsilon_{l}+\epsilon_{k}=\mathbf{a}}}(k l)^{\frac{4}{3}}\right)^{2} \leq C\left(z_{1} z_{2}\right)^{2}[l(\mathbf{a})]^{2} \sum_{\substack{l, k \\ \epsilon_{l}+\epsilon_{k}=\mathbf{a}}}(k l)^{\frac{8}{3}}
$$

where $l(\mathbf{a})$ is the number of nonzero elements of a. By (3.8), we have

$$
\begin{aligned}
\sum_{\mathbf{a} \in \mathbf{A}} \mathbf{a} ! \xi(\mathbf{a})(2 \mathbb{N})^{-p \mathbf{a}} & \leq C\left(z_{1} z_{2}\right)^{2} \sum_{\mathbf{a} \in \mathbf{A}} \mathbf{a} ![l(\mathbf{a})]^{2} \sum_{\substack{l, k \\
\epsilon_{l}+\epsilon_{k}=\mathbf{a}}}(k l)^{\frac{8}{3}}(2 \mathbb{N})^{-q \mathbf{a}} \\
& =C\left(z_{1} z_{2}\right)^{2} \sum_{l, k}\left(\epsilon_{l}+\epsilon_{k}\right) !\left[l\left(\epsilon_{l}+\epsilon_{k}\right)\right]^{2}(k l)^{\frac{8}{3}}(2 \mathbb{N})^{-q\left(\epsilon_{l}+\epsilon_{k}\right)} \\
& \leq 2^{3-2 q} C\left(z_{1} z_{2}\right)^{2}\left(\sum_{k} k^{\left(\frac{8}{3}\right)-q}\right)^{2}<\infty, \text { for } q>\frac{11}{3}
\end{aligned}
$$

We denote by $J_{B^{H}}(z)$ the Wick integral in Lemma 2. 
Theorem 2. Let $\phi: R^{2} \rightarrow(\mathbb{S})^{*}$ be a process such that $\phi(a * b) \diamond W^{H}(a) \diamond W^{H}(b)$ is dadb-integrable in $(\mathbb{S})^{*}$. Then $\phi(a) \diamond \partial_{1} B^{H}(a) \diamond \partial_{2} B^{H}(a)$ is da-integrable in $(\mathbb{S})^{*}$ and it holds

$$
\int_{R_{z} \unlhd_{1} R_{z}} \phi(a * b) d B^{H}(a) d B^{H}(b)=\int_{R_{z}} \phi(a) d J_{B^{H}}(a),
$$

where $J_{B^{H}}(z)=\int_{R_{z}} \partial_{1} B^{H}(a) \diamond \partial_{2} B^{H}(a) d a$.

Proof: By the property of Wick product, we have that $\phi(a) \diamond \partial_{1} B^{H}(a) \diamond \partial_{1} B^{H}(a) \in(\mathbb{S})^{*}$. For any $F \in(\mathbb{S})$, it follows from the definition of the $(\mathbb{S})^{*}$-integral and assumption that

$$
\ll \int_{R_{z}} \phi(a) d J_{B^{H}}(a), F \gg_{(\mathbb{S})^{*},(\mathbb{S})}=\int_{R_{z}} \ll \phi(a) \diamond \partial_{1} B^{H}(a) \diamond \partial_{1} B^{H}(a), F \gg_{(\mathbb{S})^{*},(\mathbb{S})} d a .
$$

Note that

$$
\partial_{1} B^{H}(a)=\int_{0}^{a_{2}} W^{H}(a * b) d b_{2} \quad \text { and } \quad \partial_{2} B^{H}(a)=\int_{0}^{a_{1}} W^{H}(b * a) d b_{1} .
$$

Therefore, the right-hand side of the Equation (3.10) equals

$$
\begin{aligned}
& \int_{R_{z}} \ll \phi(a) \diamond \partial_{1} B^{H}(a) \diamond \partial_{1} B^{H}(a), F \gg_{(\mathbb{S})^{*},(\mathbb{S})} d a \\
& =\int_{R_{z}} \ll \phi(a) \diamond\left(\int_{0}^{a_{2}} W^{H}(a * b) d b_{2}\right) \diamond\left(\int_{0}^{a_{1}} W^{H}(b * a) d b_{1}\right), F \gg_{(\mathbb{S})^{*},(\mathbb{S})} d a \\
& =\int_{R_{z}} \ll \int_{0}^{a_{1}} \int_{0}^{a_{2}} \phi(a) \diamond W^{H}(a * b) \diamond W^{H}(b * a) d b_{1} d b_{2}, F \gg_{(\mathbb{S})^{*},(\mathbb{S})} d a .
\end{aligned}
$$

By Fübini theorem, the last integral in (3.11) can be written as

$$
\int_{a_{1}=0}^{z_{1}} \int_{b_{2}=0}^{z_{2}} \int_{b_{1}=0}^{a_{1}} \int_{a_{2}=b_{2}}^{z_{2}} \ll \phi(a) \diamond W^{H}(a * b) \diamond W^{H}(b * a), F \gg_{(\mathbb{S})^{*},(\mathbb{S})} d b_{1} d a_{2} d a_{1} d b_{2} .
$$

By changing the role of $a_{2}$ and $b_{2}$ in the integral of (3.12), we obtain that (3.12) equals

$$
\begin{aligned}
& \int_{a_{1}=0}^{z_{1}} \int_{a_{2}=0}^{z_{2}} \int_{b_{1}=0}^{a_{1}} \int_{b_{2}=a_{2}}^{z_{2}} \ll \phi(a * b) \diamond W^{H}(a) \diamond W^{H}(b), F \gg_{(\mathbb{S})^{*},(\mathbb{S})} d a d b \\
& =\int_{a_{1}=0}^{z_{1}} \int_{a_{2}=0}^{z_{2}} \int_{b_{1}=0}^{a_{1}} \int_{b_{2}=a_{2}}^{z_{2}} \ll \phi(a * b) \diamond W^{H}(a) \diamond W^{H}(b), F \gg_{(\mathbb{S})^{*},(\mathbb{S})} d a d b \\
& =\int_{R_{z} \times R_{z}} \ll \phi(a * b) \mathbf{1}_{\left[b \unlhd_{1} a\right]} \diamond W^{H}(a) \diamond W^{H}(b), F \gg_{(\mathbb{S})^{*},(\mathbb{S})} d a d b .
\end{aligned}
$$

From (3.11) and (3.13), the process $\phi(a) \diamond \partial_{1} B^{H}(a) \diamond \partial_{2} B^{H}(a)$ is $d a$-integrable in (S)* and the Equation (3.9) holds.

We consider the third and fourth integrals in (1.1).

Theorem 3. (i) Let $\phi: R^{2} \rightarrow(\mathbb{S})^{*}$ be a process such that $\phi(a * b) \diamond W^{H}(a)$ is dadb-integrable in $(\mathbb{S})^{*}$. Then $\phi(a) \diamond \partial_{1} B^{H}(a)$ is da-integrable in $(\mathbb{S})^{*}$ and it holds

$$
\int_{R_{z} \unlhd_{1} R_{z}} \phi(a * b) d a d B^{H}(b)=\int_{0}^{z_{2}} \int_{h_{z^{*} a}} a_{1} \phi(a) \partial_{1} B^{H}(a) d a_{2} .
$$


(ii) Let $\phi: R^{2} \rightarrow(\mathbb{S})^{*}$ be a process such that $\phi(a * b) \diamond W^{H}(b)$ is dadb-integrable in $(\mathbb{S})^{*}$. Then $\phi(a) \circ \partial_{2} B^{H}(a)$ is da-integrable in $(\mathbb{S})^{*}$ and it holds

$$
\int_{R_{z} \unlhd_{1} R_{z}} \phi(a * b) d B^{H}(a) d b=\int_{0}^{z_{1}} \int_{v_{a * z}} a_{2} \phi(a) \partial_{2} B^{H}(a) d a_{1} .
$$

Proof: By the property of the Wick product, we get $\phi(a) \diamond \partial_{1} B^{H}(a) \in(\mathbb{S})^{*}$. Since $\phi(a * b) \mathbf{1}_{\left[b \unlhd_{1} a\right]^{\circ}} \diamond W^{H}(a)$ is $d a d b$-integrable in $(\mathbb{S})^{*}$, we have that for any $F \in(\mathcal{S})^{*}$,

$$
\begin{aligned}
& \int_{R_{2} \times R_{z}} \mathbb{1}_{\left[b \unlhd_{1} a\right]} \ll \phi(a * b) \diamond W^{H}(a), F \gg_{(\mathbb{S})^{*},(\mathbb{S})} d a d b \\
& =\int_{R_{2}} \int_{a_{2}=0}^{b_{2}} \int_{b_{1}=0}^{a_{1}} \ll \phi(a * b) \diamond W^{H}(a), F \gg_{(\mathbb{S})^{*},(\mathbb{S})} d a_{2} d b_{1} d a_{1} d b_{2} \\
& =\int_{0}^{z_{2}}\left(\int_{0}^{z_{1}} a_{1} \ll \phi(a) \diamond \partial_{1} B^{H}(a), F \gg_{(\mathbb{S})^{*},(\mathbb{S})} d a_{1}\right) d a_{2} \\
& =\ll \int_{0}^{z_{2}} \int_{h_{z_{*}}} a_{1} \phi(a) \diamond \partial_{1} B^{H}(a) d a_{1} d a_{2}, F \gg_{(\mathbb{S})^{*},(\mathbb{S})} \\
& =\ll \int_{0}^{z_{2}} \int_{h_{l_{*} * a}} a_{1} \phi(a) \partial_{1} B^{H}(a) d a_{2}, F \gg_{(\mathbb{S})^{*},(\mathbb{S})} .
\end{aligned}
$$

Since (3.16) holds for all $F \in(\mathcal{S})^{*}$, the equality (3.14) follows. By using the same arguments as for the proof of (3.14), we can show that the Equation (3.15) holds.

Remark 2. The new integrals defined in this paper can be used to derive Ito formula for fractional Brownian sheet. Let $f \in C^{4}(R)$ be a function such that $f\left(B^{H}(a * b)\right)$ satisfy the conditions in Theorem 2 and 3 . Then Itô formula given in Kim and Jeon (2006) can be represented as follows:

$$
\begin{aligned}
f\left(B^{H}(z)\right)= & f(0)+\int_{R_{z}} f^{\prime}\left(B_{a}^{H}\right) d B^{H}(a)+2 H \int_{R_{z}} f^{\prime \prime}\left(B^{H}(a)\right) a^{2 H-1} d a \\
& +\int_{R_{z}} f^{\prime \prime}\left(B^{H}(a)\right) d J_{H}(a) \\
& +H_{2} \int_{0}^{z_{2}} a_{2}^{2 H_{2}-1} \int_{h_{z * a}} a_{1}^{2 H_{1}} f^{(3)}\left(B^{H}(a)\right) \partial_{1} B^{H}(a) d a_{2} . \\
& +H_{1} \int_{0}^{z_{1}} a_{1}^{2 H_{1}-1} \int_{v_{a * z}} a_{2}^{2 H_{2}} f^{(3)}\left(B^{H}(a)\right) \partial_{2} B^{H}(a) d a_{1} \\
& +2 H \int_{R_{z}} f^{(4)}\left(B^{H}(a)\right) a^{4 H-1} d a .
\end{aligned}
$$

\section{References}

Biagini, F., Øksendal, B., Sulem, A. and Wallner, N. (2004). An introduction to white noise theory and Malliavin calculus for fractional Brownian motion, Proceedings: Mathematical, Physical and Engineering Sciences, 460, 347-372.

Cairoli, R. and Walsh, J. B. (1975). Stochastic integrals in the plane, Acta Mathematica, 134, 111183. 
Elliott, R. J. and van der Hoek, J. (2003). A general fractional white noise theory and applications to finance, Mathematical Finance, 13, 301-339.

Holden, H., Øksendal, B., Uboe, J. and Zhang, T. (1996). Stochastic Partial Differential Equations, Birkhauser, Boston.

Hu, Y., Øksendal, B. and Zhang, T. (2004). General fractional multiparameter white noise theory and stochastic partial differential equations, Communications in Partial Differential Equations, 29, $1-23$.

Kim, Y. T. (2006). An Itô formula of generalized functionals and local time for fractional Brownian sheet, Stochastic Analysis and Applications, 24, 973-997.

Kim, Y. T. (2009). A note on the differentiation formula in Stratonovich type for a fractional Brownian sheet, Journal of the Korean Statistical Society, 38, 259-265.

Kim, Y. T. and Jeon, J. W. (2006). An Itô formula for a fractional Brownian sheet with arbitrary Hurst parameters, Proceedings of the American Mathematical Society, 134, 3677-3683.

Kim, Y. T., Jeon, J. W. and Park, H. S. (2008). Various types of stochastic integrals with respect to fractional Brownian sheet and their applications, Journal of Mathematical Analysis and Applications, 341, 1382-1398.

Kim, Y. T., Jeon, J. W. and Park, H. S. (2009). Differentiation formula in Stratonovich version for fractional Brownian sheet, Journal of Mathematical Analysis and Applications, 359, 106-125.

Kim, Y. T. and Park, H. S. (2009). Stratonovich calculus with respect to fractional Brownian sheet, Stochastic Analysis and Applications, 27, 1-22.

Kim, Y. T. and Rhee, J. (2008). A note on Itô formula for a fractional Brownian sheet with Hurst parameters $H_{1}, H_{2} \in(0,1)$, Journal of the Korean Statistical Society, 37, 349-354.

Tudor, C. A. and Viens, F. G. (2003). Itô Formula and Local Time for the Fractional Brownian Sheet, Electronic Journal of Probability, 8, 1-31. 